\title{
Transaminases are Potential Biomarkers of Disease Severity in COVID-19 Patients: A Single- Center Experience
}

Pravallika Chadalavada ${ }^{1}$, Vinay Padbidri ${ }^{2}$, Rajat Garg ${ }^{3}$, Mohammad Alomari ${ }^{3}$, Arslan Babar $^{2}$, Tariq Kewan ${ }^{2}$, Keerat R. Ahuja ${ }^{3}$, Jose Contreras ${ }^{4}$, Mohammed J. Al-Jaghbeer ${ }^{5}$, Madhusudhan R. Sanaka ${ }^{6}$

1. Internal Medicine, Cleveland Clinic, Cleveland, USA 2. Internal Medicine, Cleveland Clinic Fairview Hospital, Cleveland, USA 3. Internal Medicine, Cleveland Clinic Foundation, Cleveland, USA 4. Critical Care Medicine, Cleveland Clinic Foundation, Cleveland, USA 5. Pulmonary and Critical Care, Cleveland Clinic, Cleveland, USA 6. Gastroenterology and Hepatology, Cleveland Clinic Foundation, Cleveland, USA

Corresponding author: Pravallika Chadalavada, pravalikasharma19@gmail.com

\section{Abstract}

Background: Considering the rapid spread of severe acute respiratory syndrome coronavirus 2 (SARS-CoV2), the clinical implications of gastrointestinal (GI) and hepatic manifestations of coronavirus disease 2019 (COVID-19) in the U.S. population require analysis.

Methods: We retrospectively reviewed all adult patients with COVID-19 admitted to our facility. Patients were divided into two groups based on the presence of GI symptoms and transaminitis at presentation. Univariable analysis was performed to assess the differences between study groups. Kruskal-Wallis and Pearson's chi-square tests were used to compare the median of continuous and categorical variables, respectively. Multivariate logistic regression analysis was performed to identify predictors of mechanical ventilation, cytokine release syndrome (CRS), and mortality after adjusting for baseline variables.

Results: A total of 84 patients were analyzed. After adjusting for baseline comorbidities, presence of GI symptoms (aOR, adjusted odds ratio $4.2,95 \% \mathrm{CI}, 1.17-15.60, \mathrm{p}=0.03$ ) and transaminitis on admission (aOR $5.69,95 \%$ CI, 1.47-21.99, $\mathrm{p}=0.01$ ) were associated with CRS. Transaminitis on admission and elevated total bilirubin during hospitalization were associated with an increased need for mechanical ventilation (aOR 6.17, 95\% CI, 1.49-25.44, $\mathrm{p}=0.02$ and aOR 7.29, 95\% CI, 1.73-30.75, $\mathrm{p}=0.007$, respectively). An elevated aspartate aminotransferase (AST) on admission (aOR 13.41, 95\% CI, 1.08-165.69, $\mathrm{p}=0.04$ ) and elevated total bilirubin during hospitalization (aOR 82.68, 95\% CI, 1.67-4074.8, $\mathrm{p}=0.02$ ) were independently associated with an increased risk of mortality in COVID-19 patients.

Conclusion: COVID-19 patients with transaminitis on admission had a higher risk of requiring mechanical ventilation and developing CRS. Patients with elevated AST on admission and elevated total bilirubin had higher mortality. Patients with GI symptoms did not have worse outcomes.

Review began 10/10/2020 Review ended 11/12/2020 Published 11/18/2020

(c) Copyright 2020

Chadalavada et al. This is an open access article distributed under the terms of the Creative Commons Attribution License CC-BY 4.0., which permits unrestricted use, distribution, and reproduction in any medium, provided the original author and source are credited.
Categories: Internal Medicine

Keywords: sars- cov2, coronavirus disease-2019, gastrointestinal, hepatic manifestations, outcomes

\section{Introduction}

The first outbreak of novel coronavirus (severe acute respiratory syndrome coronavirus 2, SARS-CoV2) occurred in Hubei province, Wuhan, China, in December 2019. Soon after, this virus has rampantly spread across the globe, and the coronavirus disease 2019 (COVID-19) was declared a public health emergency of international concern by the World Health Organization (WHO) on 30th January 2020. As of May 2020, a total of 1,385,834 laboratory-confirmed cases have been documented in the United States. Although the SARS-CoV2 is predominantly a respiratory virus, there is an ongoing investigation on the degree of gastrointestinal (GI) and hepatic involvement of this novel virus.

Emerging studies have highlighted that the angiotensin-converting enzyme 2 (ACE2) receptors not only act as potential binding sites for the SARS-CoV2 but also contribute to enhancing the progression of thrombotic and inflammatory processes [1]. These receptors are also found abundantly in the GI and biliary tract, thus raising suspicion for the GI epithelium and cholangiocytes to be additional sites of viral replication [2]. Mounting evidence from a few studies confirms the GI tropism of SARS-CoV2 by detection of this virus in stool samples of affected patients, thus providing a potential explanation for the GI symptoms, recurrence, and transmission from fecal shedding [3]. These findings were further reaffirmed by another study which reported that the SARS-CoV2 RNA was detected in the stool samples of $83.3 \%$ of patients with a mild infection for up to a month [4].

A study from Zhejiang province, China reported that $11.4 \%$ of the patients who tested positive for COVID-19 
had at least one GI symptom (nausea, vomiting, or diarrhea) at presentation [5]. Strikingly, a significantly higher percentage of patients with GI symptoms developed severe disease when compared to those without GI symptoms. Additionally, 14.8\%-78\% of the patients with COVID-19 also had abnormal alanine aminotransferase (ALT) and aspartate aminotransferase (AST) accompanied by mildly elevated bilirubin level on admission [6]. Recent studies have suggested that the presence of liver injury on presentation was associated with a significantly higher rate of ICU admission and death [7-8].

Given the rapid spread and high pathogenic capacity of SARS-CoV2 infection, we determined that a more detailed analysis of the clinical characteristics, severity, and implications of GI and hepatic manifestations of COVID-19 is required. Additionally, we also aimed to evaluate the trend of elevation of liver enzymes during hospitalization and compare the overall outcomes among patients with and without GI/hepatic manifestations.

\section{Materials And Methods \\ Patients}

We retrospectively reviewed the electronic medical records of all patients who were diagnosed with COVID19 and consecutively admitted to Cleveland Clinic Fairview Hospital between March 13th, 2020 and May 1st, 2020. All patients above the age of 18 years with a positive reverse-transcriptase-polymerase-chain-reaction (RT-PCR) assay for SARS-CoV2 at admission were included in this study. This study was approved by the institutional review board of Cleveland Clinic.

\section{Study variables and assessments}

We retrospectively collected the following data on all our included patients: age, sex, race and ethnic group, smoking status, comorbidities, clinical symptoms, first recorded vital signs on presentation, first recorded inpatient laboratory tests, oxygen requirement, diagnostic workup, treatment received, complications, and outcomes.

\section{Evaluation of gastrointestinal symptoms}

All patients with at least one of the following symptoms, such as anorexia, nausea, vomiting, diarrhea, or abdominal pain at presentation, were considered positive for GI symptoms. We recorded each of these symptoms on the first day of presentation to offset the effect of antibiotics or antivirals, which are usually started during hospitalization. Diarrhea was defined by the presence of more than three loose stools per day. Patients underwent additional testing with stool cultures, stool enteric panel, and stool PCR for Clostridium difficile to evaluate for other causes of diarrhea depending on food history, residence, occupational exposure, recent and remote travel, and antibiotic use.

\section{Evaluation of transaminitis}

All patients with at least one of the following: elevated ALT or AST > 1 x upper limit of normal (ULN) were considered to have transaminitis. All the included patients had normal baseline liver function tests except one patient with liver cirrhosis who had fluctuating liver enzymes over the past several years. These laboratory parameters were recorded on admission, precluding the influence of other medical therapy and external factors. We also collected the peak AST, ALT, alkaline phosphatase (ALP), total bilirubin, and albumin levels during hospitalization to evaluate the trend of elevation of liver enzymes.

\section{Treatment for COVID-19}

On admission, all patients with COVID-19 were started on hydroxychloroquine with a loading dose of 400 $\mathrm{mg}$ twice daily, followed by $200 \mathrm{mg}$ twice daily for additional five days and azithromycin $500 \mathrm{mg}$ per day for five days, unless contraindicated. An electrocardiogram was obtained for each patient to check the corrected QT interval at baseline. Tocilizumab was given to COVID-19 patients at the discretion of an infectious disease specialist, in the presence of hypoxia, lung infiltrates on chest radiography, elevated inflammatory biomarkers (either C-reactive protein $>3 \mathrm{~g} / \mathrm{dL}$ or ferritin $>400 \mathrm{ng} / \mathrm{mL}$ ), and concern for clinical deterioration. Tocilizumab was administered as a single dose of $400 \mathrm{mg}$, and it was given as a 60-minute single IV infusion. Tocilizumab was not given to patients with confirmed or suspected bacterial or fungal infections, platelets count $<100,000 / \mathrm{mm} 3$, neutrophil count $<2000 / \mathrm{mm} 3$, and ALT or AST more than five times the ULN range (200 IU/L).

\section{Outcomes}

Primary outcomes of interest were ICU admission, development of circulatory shock requiring vasopressor support, development of acute respiratory distress syndrome (ARDS), development of cytokine release syndrome (CRS), development of acute kidney injury (AKI) requiring renal replacement therapy, need for mechanical ventilation, and mortality. Secondary outcomes of interest were the hospital length of stay, deep vein thrombosis (DVT), duration of vasopressor support, and duration of mechanical ventilation. 


\section{Cureus}

\section{Statistical analysis}

Continuous variables were presented as median (interquartile range) and categorical variables as counts and frequency. Univariable analysis was performed to assess differences between study groups. Kruskal-Wallis test was used to compare medians of continuous variables. Pearson's chi-square tests were used to compare categorical variables. Multivariate logistic regression analysis was performed to identify predictors of mechanical ventilation, CRS, and mortality after adjusting for baseline variables. All statistical analyses were performed using the SPSS software (IBM Corporation, USA). A p-value $<0.05$ was considered statistically significant.

\section{Results}

\section{Baseline characteristics}

\section{Patients With and Without GI Symptoms}

A total of 84 patients were included in the analysis. Forty-four (52.3\%) patients had GI symptoms. The most common GI symptom was diarrhea $(n=29)$, followed by nausea and vomiting $(n=18)$, loss of appetite $(n=12)$, and abdominal pain $(\mathrm{n}=1)$. Patients with GI symptoms were significantly younger (median age: 60 years) as compared to patients without GI symptoms (median age: 68 years, $\mathrm{p}=0.01$ ). There were a significantly higher number of patients with GI symptoms in the age group of 40-64 years than those without GI symptoms (56.8\% vs. $32.5 \%$, $\mathrm{p}=0.02$ ). There were no significant differences in gender, race distribution, and smoking history between the two groups. There was no difference in baseline comorbidities between both groups, including hypertension, diabetes, chronic lung disease, chronic kidney disease, obstructive sleep apnea, atrial fibrillation, asthma, and liver cirrhosis. Majority of the patients had fever (65.4\%) on admission. The distribution of abnormal vitals, including fever, heart rate $\geqslant 100 \mathrm{bpm}$, and respiratory rate $\geqslant 20$ breaths per minute were also similar in both groups. Table 1 summarizes all the baseline characteristics of both groups with and without GI symptoms.

\begin{tabular}{|c|c|c|c|}
\hline Characteristic & Patients without GI symptoms $(n=40)$ & Patients with GI symptoms ( $=44)$ & p-Value \\
\hline Median age (IQR) - year & $68.0(22)$ & $60.0(22)$ & 0.01 \\
\hline Age 18-39 & $1(2.5)$ & $4(9.1)$ & 0.2 \\
\hline Age $40-64$ & $13(32.5 \%)$ & $25(56.8 \%)$ & 0.02 \\
\hline$>=65$ & $26(65 \%)$ & $15(34.1 \%)$ & 0.005 \\
\hline Male sex - no. (\%) & 27 (67.5\%) & $25(56.8 \%)$ & 0.31 \\
\hline \multicolumn{4}{|l|}{ Race-no. (\%) } \\
\hline Caucasian & $26(65.0 \%)$ & $33(75.0 \%)$ & 0.57 \\
\hline African American & $6(15.0 \%)$ & $4(9.1 \%)$ & \\
\hline Other & $8(20.0 \%)$ & 7 (15.9\%) & \\
\hline BMI $\left(\mathrm{kg} / \mathrm{m}^{2}\right)$ & $30.3(10.7)$ & 29.7 (8.9) & 0.85 \\
\hline Current smoker - no. (\%) & $0(0.0 \%)$ & $3(6.8 \%)$ & 0.08 \\
\hline Former smoker - no. (\%) & 19 (47.5\%) & $16(37.2 \%)$ & 0.34 \\
\hline \multicolumn{4}{|c|}{ Comorbid conditions - no. (\%) } \\
\hline HTN & $26(65.0 \%)$ & $30(68.2 \%)$ & 0.75 \\
\hline DM & $14(35.0 \%)$ & $16(36.4 \%)$ & 0.89 \\
\hline COPD & $6(15.0 \%)$ & $3(6.8 \%)$ & 0.22 \\
\hline CKD & $5(12.5 \%)$ & $4(9.1 \%)$ & 0.73 \\
\hline CAD & $8(20.0 \%)$ & $9(20.5 \%)$ & 0.95 \\
\hline Asthma & $8(20.0 \%)$ & $10(22.7 \%)$ & 0.76 \\
\hline CVA & $1(2.5 \%)$ & $0(0)$ & 0.47 \\
\hline OSA & $6(15.0 \%)$ & 4 (9.1\%) & 0.5 \\
\hline Atrial fibrillation & 2 (5.0\%) & 1 (2.3\%) & 0.5 \\
\hline
\end{tabular}




\section{Cureus}

\begin{tabular}{|c|c|c|c|}
\hline Liver cirrhosis & $5(12.5 \%)$ & $3(6.8 \%)$ & 0.37 \\
\hline ACE/ARB use & $12(30 \%)$ & $21(47.7 \%)$ & 0.09 \\
\hline NSAID use & $4(10 \%)$ & $10(22.7 \%)$ & 0.11 \\
\hline \multicolumn{4}{|l|}{ Symptoms on admission - no. (\%) } \\
\hline Fever & $24(60.0 \%)$ & $31(70.5 \%)$ & 0.31 \\
\hline Fatigue & $19(47.5 \%)$ & $23(52.3 \%)$ & 0.66 \\
\hline Sore throat & $3(7.5 \%)$ & $4(9.1 \%)$ & 0.19 \\
\hline Cough & 21 (52.5\%) & $32(72.7 \%)$ & 0.06 \\
\hline SOB & 25 (62.5\%) & $32(72.7 \%)$ & 0.35 \\
\hline AMS & $5(12.5 \%)$ & $5(11.4 \%)$ & 0.87 \\
\hline \multicolumn{4}{|l|}{ Vitals on admission } \\
\hline Temperature, median (IQR) $-{ }^{0} \mathrm{~F}$ & $99.0(1.7)$ & $99.0(2.2)$ & 0.74 \\
\hline Heart rate >100 beats per $\min -$ no. $(\%)$ & $9(22.5 \%)$ & $12(27.3 \%)$ & 0.8 \\
\hline Respiratory rate $>20$ breaths per minute - no. $(\%)$ & $23(57.5 \%)$ & 24 (54.5\%) & 0.7 \\
\hline
\end{tabular}

\section{Patients With and Without Transaminitis (AST or ALT > 40 IU/L)}

In patients with and without transaminitis, the baseline features, including age, race, and comorbidities, were similar in both groups. There was a significantly higher number of male patients with transaminitis than without transaminitis $(79.5 \%$ vs. $42.5 \%, \mathrm{p}=0.0001)$. All the baseline characteristics of patients with and without transaminitis are shown in Table 2. 


\section{Cureus}

\begin{tabular}{|c|c|c|c|}
\hline Characteristic & Patients without transaminitis $\quad(n=40)$ & Patients with transaminitis $(n=44)$ & p-Value \\
\hline Median age (IQR) - years & $64.0(21)$ & $63.5(26)$ & 0.96 \\
\hline Male - no. (\%) & $17(42.5 \%)$ & $35(79.5 \%)$ & 0.001 \\
\hline Caucasian race - no. (\%) & $28(70 \%)$ & $31(70.5 \%)$ & 0.98 \\
\hline BMI $\left(\mathrm{kg} / \mathrm{m}^{2}\right)$ & $30.5(10.1)$ & $29.7(8.6)$ & 0.72 \\
\hline African American - no. (\%) & $5(12.5 \%)$ & $5(11.4 \%)$ & \\
\hline Current smoker - no. (\%) & $1(2.5 \%)$ & $2(4.5 \%)$ & 0.61 \\
\hline Former smoker - no. (\%) & $19(47.5 \%)$ & $16(37.2 \%)$ & 0.34 \\
\hline \multicolumn{4}{|l|}{ Comorbidities - no. (\%) } \\
\hline HIN & $26(65.0 \%)$ & $30(68.2 \%)$ & 0.81 \\
\hline DM & $17(42.5 \%)$ & $13(29.5 \%)$ & 0.25 \\
\hline COPD & $7(17.5 \%)$ & $2(4.5 \%)$ & 0.06 \\
\hline CKI & $5(12.5 \%)$ & $4(9.1 \%)$ & 0.73 \\
\hline CAD & $9(22.5 \%)$ & $8(18.2 \%)$ & 0.62 \\
\hline Asthma & $9(22.5 \%)$ & $9(20.5 \%)$ & 0.82 \\
\hline CVA & $0(0)$ & $1(2.3 \%)$ & 0.33 \\
\hline OSA & $5(12.5 \%)$ & $5(11.4 \%)$ & 0.87 \\
\hline Atrial fibrillation & $1(2.5 \%)$ & $2(4.5 \%)$ & 0.61 \\
\hline Liver cirrhosis & $2(5.0 \%)$ & $6(13.6 \%)$ & 0.17 \\
\hline ACE/ARB USe & $13(32.5 \%)$ & $20(45.5 \%)$ & 0.22 \\
\hline NSAID use & $5(12.5 \%)$ & $9(20.5 \%)$ & 0.32 \\
\hline \multicolumn{4}{|l|}{ Vitals at presentation } \\
\hline Temperature, median (IQR) $-{ }^{0} \mathrm{~F}$ & $99.05(2.9)$ & $99.05(1.3)$ & 0.71 \\
\hline Heart rate $>100$ beats per $\min -$ no. $(\%)$ & $8(20 \%)$ & $13(29.5 \%)$ & 0.45 \\
\hline $\begin{array}{l}\text { Respiratory rate > } 20 \text { breaths per min - no. } \\
(\%)\end{array}$ & $22(55 \%)$ & $25(56.8 \%)$ & 0.86 \\
\hline
\end{tabular}

\section{TABLE 2: Baseline demographics in patients with and without transaminitis.}

HTN, hypertension; DM, diabetes mellitus; COPD, chronic obstructive pulmonary disease; CKI, chronic kidney disease; CAD, coronary artery disease; CVA, cerebrovascular accident; OSA, Obstructive sleep apnea; ACE/ARB, angiotensin converting enzyme/angiotensin II receptor blocker; NSAID, nonsteroidal anti-inflammatory drug

\section{Laboratory and imaging parameters \\ Patients With and Without GI Symptoms}

The median white cell count was 5,800 and 6,700 per mm3 in patients with and without GI symptoms, respectively ( $\mathrm{p}=0.17$ ). The incidence of lymphopenia, thrombocytopenia, and elevated creatinine on admission was also similar in both groups. The median AST, ALT, and total bilirubin were also identical in both groups. The median CRP was 7.9 and $10.5 \mathrm{mg} / \mathrm{dL}$ in patients with and without GI symptoms, respectively ( $\mathrm{p}=0.39$ ). The distribution of ferritin, fibrinogen, interleukin-6, and D-dimer was not significantly different in both groups. There was also no significant difference in median peak AST, ALT, and total bilirubin between both groups. There was only one case each of concurrent influenza and respiratory syncytial virus in our study population. On imaging, majority (77.3\%) of the patients had bilateral infiltrates, and imaging findings were also similar in these groups. Table 3 summarizes all the laboratory and imaging parameters of patients with and without GI symptoms. 


\section{Cureus}

\section{Factor}

Laboratory Findings on Admission

White cell count, median (IQR) - per $\mathrm{mm}^{3}$

Lymphocyte count, median (IQR) - per $\mathrm{mm}^{3}$

Lymphocyte $<1,500$, median (IQR) - per $\mathrm{mm}^{3}$

Platelet count, median (IQR) - per $10^{9} / \mathrm{L}$

Serum creatinine, median (IQR) - mg/dL

Aspartate aminotransferase (AST), median (IQR) -

U/L

Alanine aminotransferase (ALT), median (IQR) - U/L

Total bilirubin, median (IQR) - mg/dl

Troponin, median (IQR) - ng/dl

Troponin $>0.03 \mathrm{ng} / \mathrm{mL}$ - positive/total no. (\%)

IL-6, median (IQR) - pg/mL

CRP, median (IQR) - mg/dL

Ferritin, median (IQR) - ng/mL

Fibrinogen, median (IQR) - mg/dL

D-dimer, median (IQR) - ng/mL

Peak labs during hospitalization

Peak AST, median (IQR) - U/L

AST >1x ULN - no. (\%)

Peak ALT, median (IQR) - U/L

ALT >1x ULN - no. (\%)

Peak ALP, median (IQR) - U/L

ALP >123 IU/L - no. (\%)

Peak Total Bilirubin, median (IQR) - U/L

Total bilirubin $>1.3 \mathrm{mg} / \mathrm{dL}-$ no. $(\%)$

Co-infection - no. (\%)

Influenza PCR

RSV PCR

CXR findings - no. (\%)

Bilateral infiltrates

Unilateral infiltrates

No infiltrates
Patients without GI symptoms ( $n=40)$

Patients with GI symptoms ( $n=44)$

p-Value

$6.7(4.9)$

$5.8(2.8)$

0.17

$1.08(0.77)$

$0.96(0.91)$

0.54

$31(77.5 \%)$

$33(75 \%)$

0.78

221.5 (115.5)

$191.0(101.3)$

0.36

$1.03(0.67)$

$0.96(0.62)$

0.59

37 (41)

38.5 (26)

0.7

28.5 (36)

$0.5(0.4)$

$23.0(30)$

0.43

$0.5(0.4)$

0.86

$0.01(0.02)$

$0.01(0.01)$

0.07

$9(22.5 \%)$

5 (11.4\%)

0.17

$19.0(53.6)$

$29.0(52.8)$

0.65

10.55 (12.6)

7.9 (9.5)

0.39

652.2 (560.1)

602.1 (841.6)

0.68

538 (287)

598 (283)

0.73

1190 (2540)

985 (1921)

0.87

$57.0(73)$

$68.0(87.5)$

0.56

$25(62.5 \%)$

$31(70.5 \%)$

0.49

$54.0(68)$

$68.0(97)$

0.49

$24(60.0 \%)$

$30(68.2 \%)$

0.43

$90.5(43)$

83.0 (49)

0.76

7 (17.5\%)

$10(22.7 \%)$

0.55

$0.55(0.7)$

$0.7(0.8)$

0.65

$8(20.0 \%)$

$10(22.7 \%)$

0.76

$0(0 \%)$

$1(2.3 \%)$

$>0.99$

$0(0 \%)$

$1(2.3 \%)$

$>0.99$

0.82

33 (75\%)

$5(11.4 \%)$

$6(13.6 \%)$

TABLE 3: Laboratory and imaging findings in patients with and without GI symptoms.

AST, aspartate aminotransferase; ALT, alanine aminotransferase; CRP, C-reactive protein; ULN, upper limit of the normal; ALP, alkaline phosphatase; PCR, polymerase chain reaction; CXR, chest X-ray; RSV PCR, respiratory syncytial virus polymerase chain reaction 


\section{Cureus}

\section{Patients With and Without Transaminitis}

In patients with transaminitis, there was no significant difference in total leukocytes count and lymphopenia as compared to patients without transaminitis. The median platelets count was significantly lower in patients with transaminitis ( $180 \mathrm{~K} / \mathrm{uL}$ vs. $265 \mathrm{~K} / \mathrm{uL}, \mathrm{p}=0.04)$. Patients with transaminitis also had significantly elevated serum creatinine $(1.01 \mathrm{mg} / \mathrm{dL}$ vs. $0.87 \mathrm{mg} / \mathrm{dL}, \mathrm{p}=0.04)$ at the time of admission. Ferritin level on admission was almost doubled and significantly elevated in patients with transaminitis (median 746 $\mathrm{ng} / \mathrm{mL}$ ) as compared to patients without transaminitis (median $370 \mathrm{ng} / \mathrm{mL}, \mathrm{p}=0.004$ ). However, other laboratory parameters, including CRP, D-dimer, interleukin-6, and fibrinogen levels, were similar in both groups. The chest X-ray findings were also not different in both groups. These results are displayed in Table 4.

\begin{tabular}{|c|c|c|c|}
\hline Factor & Patients without transaminitis $(n=40)$ & Patients with transaminitis $(n=44)$ & p-Value \\
\hline \multicolumn{4}{|l|}{ Laboratory values on admission } \\
\hline White cell count, median (IQR) - per $\mathrm{mm}^{3}$ & $6.9(4.2)$ & $5.6(2.4)$ & 0.68 \\
\hline Lymphocyte count, median (IQR) - per mm³ & $1.00(1.03)$ & $0.78(0.80)$ & 0.53 \\
\hline Lymphocyte $<1,500$, median (IQR) - per $\mathrm{mm}^{3}$ & $29(72.5 \%)$ & $35(79.5 \%)$ & 0.44 \\
\hline Platelet count, median (IQR) - per 10\%/L & $265(75.5)$ & $180(76)$ & 0.04 \\
\hline Serum creatinine, median (IQR) - mg/dl & $0.87(0.35)$ & $1.01(0.64)$ & 0.04 \\
\hline AST, median (IQR) - U/L & $27.5(12)$ & $63(41)$ & $<0.001$ \\
\hline ALT, median (IQR) - U/L & $15.5(7)$ & $48.5(34)$ & $<0.001$ \\
\hline Total bilirubin, median (IQR) - mg/dl & $0.40(0.5)$ & $0.45(0.3)$ & 0.06 \\
\hline Troponin, median (IQR) - ng/dl & $0.01(0.01)$ & $0.01(0.02)$ & 0.36 \\
\hline Interleukin-6, median (IQR) - pg/mL & 48.9 (104.1) & $29.5(51.3)$ & 0.55 \\
\hline C-reactive protein (mg/dL) & $11.9(12.08)$ & $9.2(10.4)$ & 0.37 \\
\hline Ferritin, median (IQR) - ng/mL & $370(541)$ & 746 (1161) & 0.004 \\
\hline Fibrinogen, median (IQR) - mg/mL & $627(209)$ & $548(296)$ & 0.28 \\
\hline D-dimer, median (IQR) - ng/mL & 1850 (3325) & $4834(3940)$ & 0.96 \\
\hline \multicolumn{4}{|l|}{ Peak labs during hospitalization } \\
\hline AST, median (IQR) - U/L & 40.5 (44.5) & 79.0 (77.5) & 0.004 \\
\hline ALT, median (IQR) - U/L & $38.5(62)$ & $72.0(99)$ & 0.009 \\
\hline Total bilirubin, median (IQR) - mg/dL & $0.55(0.7)$ & $0.7(0.8)$ & 0.13 \\
\hline \multicolumn{4}{|l|}{ Co-Intection - no. (\%) } \\
\hline Influenza PCR & $1(2.5 \%)$ & $0(0)$ & 0.29 \\
\hline RSV PCR & $0(0)$ & $1(2.3 \%)$ & 0.33 \\
\hline \multicolumn{4}{|l|}{ CXR findings - no. (\%) } \\
\hline Bilateral infiltrates & $3(82.5 \%$ & $32(72.7 \%)$ & 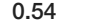 \\
\hline
\end{tabular}

\section{TABLE 4: Laboratory and imaging findings in patients with and without transaminitis.}

AST, aspartate aminotransferase; ALT, alanine aminotransferase; PCR, polymerase chain reaction; CXR, chest X-ray; RSV, respiratory syncytial virus;

\section{Outcomes}

Patients With and Without GI Symptoms 


\section{Cureus}

There were $59.5 \%(n=50)$ patients who required admission to the ICU during their hospitalization. ICU admissions were similar between both groups. Some $62 \%$ of the patients admitted to ICU required vasopressor support. Overall, vasopressor use and duration were not significantly different in both groups. The incidence of DVT was also similar in both groups. Almost half (48.8\%) of our study population had CRS. Renal replacement therapy was required in $10.7 \%$ of patients, and rates were similar between both groups. Mechanical ventilation was required in $40.4 \%(n=34)$ of patients during their hospital admission. There was no difference in the need for mechanical ventilation between both groups $(\mathrm{p}=0.93)$. The duration of mechanical ventilation was also not different in both groups ( $7 \%$ vs. $9 \%, p=0.86)$. The mortality rate in our study population was $13 \%(\mathrm{n}=11)$. Among them, six patients had no GI symptoms, and five patients had GI symptoms at presentation. There was no significant difference in mortality between both groups $(\mathrm{p}=0.62)$. These results are summarized in Table 5 .

\begin{tabular}{|c|c|c|c|}
\hline Factor & Patients without GI symptoms ( $n=40)$ & Patients with GI symptoms $(n=44)$ & p-Value \\
\hline Admission to ICU - no. (\%) & $24(60 \%)$ & $26(59.1 \%)$ & 0.93 \\
\hline Circulatory shock requiring vasopressors - no. (\%) & $15(37.5 \%)$ & $16(36.4 \%)$ & $>0.99$ \\
\hline $\begin{array}{l}\text { Mean duration of pressor support, median (IQR) - } \\
\text { day }\end{array}$ & $4(3)$ & $5(8)$ & 0.35 \\
\hline Deep vein thrombosis - no. (\%) & $2(5.0 \%)$ & $5(11.4 \%)$ & 0.43 \\
\hline Cytokine release syndrome - no. (\%) & $16(40.0 \%)$ & $25(56.8 \%)$ & 0.13 \\
\hline AKI requiring RRT use (CRRT or dialysis) - no. (\%) & $3(7.5 \%)$ & $6(13.6 \%)$ & 0.48 \\
\hline Mechanical ventilation on admission - no. (\%) & $6(15.0 \%)$ & $7(15.9 \%)$ & 0.9 \\
\hline Mechanical ventilation anytime - no. (\%) & $16(40 \%)$ & $18(40.9 \%)$ & 0.93 \\
\hline $\begin{array}{l}\text { Duration of mechanical ventilation, median (IQR) - } \\
\text { day }\end{array}$ & $11($ (n) & $9(9)$ & 0.82 \\
\hline LOS, median (IQR) - day & $10.5(13.7)$ & $7.5(8.25)$ & 0.86 \\
\hline Mortality-no. (\%) & $6(15.0 \%)$ & $5(11.4 \%)$ & 0.62 \\
\hline
\end{tabular}

\section{TABLE 5: Outcomes in patients with and without GI symptoms.}

GI, gastrointestinal; AKI, acute kidney injury; RRT, renal replacement therapy; CRRT, continuous renal replacement therapy; LOS, length of stay

\section{Patients With and Without Transaminitis}

There was no difference in the need for ICU admission, requirement, and duration of vasopressor support between both groups. There was a significantly higher number of DVT in patients with transaminitis than in patients without transaminitis $(15.9 \%$ vs. $0, p=0.01)$. The incidence of CRS was also significantly higher in patients with transaminitis $(59.1 \%)$ as compared to patients without transaminitis $(37.5 \%)(\mathrm{p}=0.04)$. The requirement of renal replacement therapy was similar in both groups. Patients with transaminitis were significantly likely to develop hypoxic respiratory failure $(86.4 \%$ vs. $65.0 \%, \mathrm{p}=0.02)$ and require mechanical ventilation $(52.3 \%$ vs. $27.5 \%, \mathrm{p}=0.02)$ as compared to patients without transaminitis. However, the duration of mechanical ventilation and length of stay in the hospital was not different between both these groups. There was a trend towards higher mortality in patients with transaminitis (18.2\%) as compared to those without transaminitis (7.5\%); however, it did not reach statistical significance $(\mathrm{p}=0.14)$ (Table $)$. 


\section{Cureus}

\begin{tabular}{|c|c|c|c|}
\hline Factor & Patients without transaminitis $(n=40)$ & Patients with transaminitis $(n=44)$ & p-Value \\
\hline Need for ICU admission -no. (\%) & $22(55.0 \%)$ & $28(63.6 \%)$ & 0.5 \\
\hline Circulatory shock requiring vasopressors - no. (\%) & $12(30 \%)$ & $19(43.2 \%)$ & 0.26 \\
\hline Mean duration of pressor support, median (IQR) - day & $5(11)$ & $2(6)$ & 0.63 \\
\hline DVT - no. (\%) & $0(0 \%)$ & $7(15.9 \%)$ & 0.008 \\
\hline CRS - no. (\%) & $15(37.5 \%)$ & $26(59.1 \%)$ & 0.04 \\
\hline AKI requiring RRT use (CRRT or dialysis) - no. (\%) & $2(5 \%)$ & $7(15.9 \%)$ & 0.1 \\
\hline AHRF requiring oxygen - no. (\%) & $26(65 \%)$ & $38(86.3 \%)$ & 0.022 \\
\hline AHRF requiring mechanical ventilation - no. (\%) & $11(27.5 \%)$ & $23(52.3 \%)$ & 0.02 \\
\hline Duration of mechanical ventilation, median (IQR) - day & $13.5(6)$ & $10.0(8)$ & 0.18 \\
\hline LOS, median (IQR) - day & $21(15.2)$ & $25.0(13.25)$ & 0.3 \\
\hline Mortality - no. (\%) & $3(7.5 \%)$ & $8(18.2 \%)$ & 0.1 \\
\hline
\end{tabular}

\section{TABLE 6: Outcomes in patients with and without transaminitis.}

DVT, deep vein thrombosis; CRS, cytokine release syndrome; AKI, acute kidney injury; RRT, renal replacement therapy; CRRT, continuous renal replacement therapy; AHRF, acute hypoxemic respiratory failure; LOS, length of stay

\section{Predictors of CRS, mechanical ventilation, and mortality}

We performed multivariate logistic regression with an adjusted odds ratio (aOR) to identify predictors of CRS, mechanical ventilation, and mortality with a particular focus on GI symptoms and transaminitis on admission and during hospitalization. We adjusted for age, gender, various comorbidities including smoking history, hypertension, diabetes mellitus, chronic kidney disease, chronic obstructive pulmonary disease, asthma, coronary artery disease, obstructive sleep apnea, atrial fibrillation, and liver cirrhosis. After adjusting for these variables, presence of GI symptoms (aOR 4.2, 95\% CI, 1.17-15.60, p=0.03), elevated AST on admission (aOR 4.7, 1.08-20.38, $\mathrm{p}=0.03$ ), transaminitis on admission (aOR 5.69, 95\% CI, 1.47-21.99, $\mathrm{p}=0.01$ ), and elevated ALT during hospitalization ( $\mathrm{aOR} 31.32$, 95\% CI, 3.75-260.94, $\mathrm{p}=0.001$ ) were significantly associated with development of CRS.

The presence of either elevated AST or transaminitis on admission was also independently associated with the need for mechanical ventilation with aOR of 6.17 (95\% CI, 1.49-25.44, p=0.02), and 9.26 (95\% CI, 2.27$37.75, \mathrm{p}=0.02$ ) respectively. An elevated ALT during hospitalization was associated with an increased risk of mechanical ventilation with aOR of 6.74 (95\% CI, 1.14-39.58, $\mathrm{p}=0.03)$. Also, total bilirubin $>1 \mathrm{x}$ ULN was also independently associated with the need for mechanical ventilation with aOR of 7.29 (95\% CI, 1.73-30.75, $\mathrm{p}=0.007$ ).

An elevated AST on admission with aOR of 13.41 (95\% CI, 1.08-165.69, p=0.04) and total bilirubin > $1 \mathrm{x}$ ULN during hospitalization with aOR of 82.68 (95\% CI, 1.67-4074.8, $\mathrm{p}=0.02)$ were independently associated with risk of death in COVID-19 patients. The results of multivariate analysis with aOR are shown in Table 7 . The predictors of CRS, mechanical ventilation, and mortality after multivariate analysis are graphically displayed in Figures 1-3, respectively. 


\section{Cureus}

\begin{tabular}{|c|c|c|c|c|c|c|}
\hline & Mechanical ventilation & & CRS & & Death & \\
\hline Factor & Adjusted OR & $p$-Value & Adjusted OR & $p$-Value & Adjusted OR & $p$-Value \\
\hline \multicolumn{7}{|l|}{ On admission } \\
\hline Gl symptoms & $1.53(0.47,4.94)$ & 0.47 & $4.27(1.17,15.60)$ & 0.03 & $0.57(0.08,3.96)$ & 0.57 \\
\hline AST > $1 \times$ ULN & $6.17(1.49,25.44)$ & 0.02 & $4.70(1.08,20.38)$ & 0.03 & $13.41(1.08,165.69)$ & 0.04 \\
\hline ALT $>1 \times$ ULN & $0.93(0.17,4.98)$ & 0.93 & $0.76(0.13,4.15)$ & 0.75 & $0.53(0.03,9.16)$ & 0.66 \\
\hline Transaminitis & $9.26(2.27,37.75)$ & 0.02 & $5.69(1.47,21.99)$ & 0.01 & $9.34(0.78,111.21)$ & 0.07 \\
\hline Total bilirubin $>1 \times$ ULN & $0.48(0.07,3.05)$ & 0.44 & $0.17(0.02,1.19)$ & 0.08 & $1.04(0.03,29.73)$ & 0.97 \\
\hline \multicolumn{7}{|l|}{ During hospitalization } \\
\hline AST > $1 \times$ ULN & $1.37(0.19,9.91)$ & 0.75 & $0.92(0.14,5.81)$ & 0.93 & $0.79(0.04,15.03)$ & 0.88 \\
\hline ALT $>1 \times$ ULN & $6.74(1.14,39.58)$ & 0.03 & $31.32(3.75,260.94)$ & 0.001 & $0.64(0.03,10.68)$ & 0.75 \\
\hline Transaminitis & $3.36(0.57,21.26)$ & 0.17 & $3.89(0.76,19.92)$ & 0.1 & $9.34(0.78,111.21)$ & 0.07 \\
\hline Total bilirubin > $1 \times$ ULN & $7.29(1.73,30.75)$ & 0.007 & $1.43(0.38,5.41)$ & 0.59 & $82.68(1.67,4074.86)$ & 0.02 \\
\hline
\end{tabular}

TABLE 7: Multivariate logistic regression showing predictors of mechanical ventilation, CRS, and mortality.

CRS, cytokine release syndrome; GI, gastrointestinal; AST, aspartate aminotransferase; ALT, alanine aminotransferase; ULN, upper limit of the normal; OR, odds ratio

\begin{tabular}{|c|c|c|c|}
\hline Variable & & Odds Ratio $(95 \% \mathrm{CI})$ & P-value \\
\hline \multicolumn{4}{|l|}{ Death } \\
\hline Total Bilirubin $>1 \times$ ULN ${ }^{*}$ hospitalization & $\longmapsto$ & $82.68(1.67,4074.86)$ & 0.02 \\
\hline AST > $1 \times$ ULN* admission & & $13.41(1.08,165.69)$ & 0.04 \\
\hline Transaminitis > 1x ULN* admission & & $9.34(0.78,111.21)$ & 0.07 \\
\hline Total Bilirubin > $1 \times$ ULN * admission & 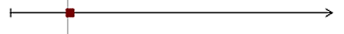 & $1.04(0.03,29.73)$ & 0.97 \\
\hline AST>1 ULN* hospitalization & $\longmapsto$ & $0.79(0.04,15.03)$ & 0.88 \\
\hline ALT>1 ULN* hospitalization & $\longmapsto$ & $0.64(0.03,10.68)$ & 0.75 \\
\hline Transaminitis > 1x ULN* hospitalization & $\longmapsto$ & $0.63(0.04,9.98)$ & 0.74 \\
\hline GI symptoms & $\longmapsto$ & $0.57(0.08,3.96)$ & 0.57 \\
\hline ALT > $1 \times$ ULN* admission & $\longmapsto$ & $0.53(0.03,9.16)$ & 0.66 \\
\hline
\end{tabular}

FIGURE 1: Forest plot of multivariate logistic regression showing predictors of mortality. 


\section{Cureus}

\begin{tabular}{|c|c|c|c|}
\hline Variable & & Odds Ratio $(95 \% \mathrm{Cl})$ & P-value \\
\hline \multicolumn{4}{|l|}{ Mechanical Ventilation } \\
\hline Transaminitis > $1 \mathrm{x} \mathrm{ULN*}$ admission & & $9.26(2.27,37.75)$ & 0.02 \\
\hline Total Bilirubin $>1 \times$ ULN* hospitalization & & $7.29(1.73,30.75)$ & 0.007 \\
\hline ALT>1 ULN* hospitalization & & $6.74(1.14,39.58)$ & 0.03 \\
\hline AST $>1$ ULN * admission & $\rightarrow$ & $6.17(1.49,25.44)$ & 0.02 \\
\hline Transaminitis > 1x ULN* hospitalization & $\longmapsto$ & $3.36(0.57,21.26)$ & 0.17 \\
\hline GI symptoms & -1 & $1.53(0.47,4.94)$ & 0.47 \\
\hline AST>1 ULN* hospitalization & & $1.37(0.19,9.91)$ & 0.75 \\
\hline ALT $>1$ ULN * admission & $\longmapsto$ & $0.93(0.17,4.98)$ & 0.93 \\
\hline Total Bilirubin > $1 \mathrm{x}$ ULN* admission & $\longrightarrow$ & $0.48(0.07,3.05)$ & 0.44 \\
\hline
\end{tabular}

FIGURE 2: Forest plot of multivariate logistic regression showing predictors of mechanical ventilation.

\begin{tabular}{|c|c|c|c|}
\hline Variable & & Odds Ratio $(95 \% \mathrm{CI})$ & P-value \\
\hline \multicolumn{4}{|l|}{ Cytokine Release Syndrome } \\
\hline ALT>1 ULN* hospitalization & & $31.32(3.75,260.94)$ & 0.001 \\
\hline Transaminitis $>1 \mathrm{x}$ ULN * admission & & $5.69(1.47,21.99)$ & 0.01 \\
\hline GI symptoms & $\rightarrow$ & $4.27(1.17,15.60)$ & 0.03 \\
\hline Transaminitis > 1x ULN * hospitaliza & & $3.89(0.76,19.92)$ & 0.1 \\
\hline AST $>1$ ULN* $^{*}$ admission & & $4.70(1.08,20.38)$ & 0.03 \\
\hline Total Bilirubin $>1 \times$ ULN* hospitaliz & & $1.43(0.38,5.41)$ & 0.59 \\
\hline AST>1 ULN * hospitalization & & $0.92(0.14,5.81)$ & 0.93 \\
\hline ALT $>1$ ULN* $^{*}$ admission & & $0.76(0.13,4.15)$ & 0.75 \\
\hline Total Bilirubin $>1 \times$ ULN* admission & -1 & $0.17(0.02,1.19)$ & 0.08 \\
\hline
\end{tabular}

FIGURE 3: Forest plot of multivariate logistic regression showing predictors of CRS.

CRS, cytokine release syndrome

\section{Discussion}

To the best of our knowledge, this is the first study comparing the clinical course and outcomes of COVID19 patients with and without gastrointestinal and hepatic manifestations in the U.S. population. Our study revealed that $52 \%$ of our patients who tested positive for SARS-CoV2 had at least one GI symptom at presentation. Our findings are in line with the studies by Pan et al. [9] and Zhang et al. [2], who reported an incidence of $50.7 \%$ and $39.6 \%$ for GI symptoms in their COVID-19 patients, respectively. Diarrhea was the most common GI symptom followed by nausea/vomiting and loss of appetite. Only one patient had abdominal pain in our study population. As such, our findings add to the evolving evidence that SARS-CoV2 might cause acute gastritis and enteritis.

Interestingly, a higher proportion of younger patients aged 40-64 years had GI symptoms when compared to more respiratory symptoms in patients aged $>65$ years. This corroborates with the observation by Lin et al. [10], where the mean age of patients with GI symptoms was 48 years. Moreover, a higher proportion of our 
patients also had elevated AST or ALT at presentation. On multivariate analysis, the presence of transaminitis on admission was associated with a higher likelihood of developing ARDS requiring mechanical ventilation and CRS. Notably, an elevated AST on admission and elevated total bilirubin during hospitalization were associated with significantly higher mortality.

Previously published studies from China reported a higher likelihood of severe or critical illness in patients with GI symptoms. In a study by Jin et al. [5], a significantly higher percentage of patients with GI symptoms required admission to the ICU, were on mechanical ventilation, and developed transaminitis during hospitalization. A recent metanalysis of 35 studies by Mao et al. [8] suggested that COVID-19 patients with GI symptoms had a higher risk of developing severe disease. Despite the high incidence of GI symptoms in our patients, their presence did not seem to have any significant impact on the overall outcomes. There was no difference in the need for mechanical ventilation or mortality among patients with and without GI symptoms, except for an increased incidence of CRS. These findings are relatively similar to a large study from New York, who suggested that the presence of GI symptoms was associated with less severe disease and had no significant impact on the disease outcomes on multivariate analysis [7]. As such, it appears that the presence of GI symptoms in the American population might not have a strong association with a worsening disease when compared to the Asian population.

In addition to the GI tract involvement, hepatic manifestations have also been commonly reported in patients infected with SARS-CoV2. Recent studies have suggested a varying degree of liver enzymes elevation in patients with COVID-19, with a reported incidence ranging from $14.8 \%$ to $78 \%$ [11-14]. In a study from China, $78 \%$ of the patients with severe disease had increased transaminase levels [15]. They further suggested a strong association with increased AST or ALT with higher mortality. The authors suggested that there seemed to be a higher prevalence of liver injury in patients with severe disease when compared to those with mild disease. Interestingly, Jin et al. [5] also found a higher incidence of transaminitis in patients with GI symptoms, which could have indirectly caused worsening disease activity. Thus, data from all these studies does highlight the fact that the occurrence of abnormal liver function tests during hospitalization for COVID-19 is quite common and clinically significant liver injury or liver failure is rare [16-18]. However, it is still unclear if the elevated transaminases during hospitalization are indeed a surrogate clinical maker for higher levels of viremia.

An article describing the risk factors associated with hepatic injury in COVID-19 patients suggested the presence of lymphopenia and high CRP level to be independent risk factors for liver enzymes abnormalities [19]. Although the underlying mechanism of liver injury remains unclear, various direct and indirect etiologies have been postulated thus far. These include the cytopathic effect of the virus [20], hypoperfusion following circulatory shock [21], presence of multiorgan failure [22], drug-induced liver injury [23], and T-cell activated inflammatory responses such as cytokine release storm from SARS-CoV2 [24]. Other less unlikely but possible etiologies are hepatic congestion secondary to the use of positive pressure ventilation [20], ischemia, and infectious myositis. As such, the presence of elevated liver enzymes during hospitalization could be multifactorial and cannot be attributed to the primary spectrum of coronavirus disease. In our study, we attempted to primarily evaluate the implications of abnormal liver enzymes that occurred at the time of diagnosis, than during treatment or hospitalization.

We evaluated for the presence of abnormal liver enzymes at presentation, thereby excluding the effect of external factors such as antiviral medications (lopinavir/ritonavir) [21], IL-6 inhibitors (tocilizumab) [25], antipyretics (acetaminophen) [6], antibiotics (macrolides, quinolones), steroids, positive pressure ventilation, etc. Additionally, the baseline comorbidities, including cirrhosis, first recorded laboratory parameters, and oxygen requirements on admission were not significantly different among our patients, thus eliminating the significant heterogeneity among our study groups. With the current emphasis on streamlining triaging efforts and healthcare resource utilization, we tried to perform a more detailed analysis of the clinical manifestations, hospital course, and outcomes of COVID-19 patients with and without GI and hepatic manifestations. Furthermore, we performed a robust statistical analysis by adjusting for comorbidities to decrease potential confounders.

Our study has several limitations, apart from having a single-center retrospective observational design. First, our findings cannot be widely generalized due to the relatively small study population and the rapidly changing demographic and clinical pattern of SARS-CoV2. Second, our study population represents our very first encounter with COVID-19 patients who were treated with medications that are currently under investigation with no clear, proven efficacy. Third, due to the short duration of our study period, we could not assess the factors associated with prolonged length of stay and readmissions in these patients. Lastly, although majority of our patients had hypertransaminasemia at presentation, we could not elucidate the primary cause of this phenomenon to guide the best treatment.

\section{Conclusions}

In conclusion, the characteristics, clinical course, and outcomes of COVID-19 patients with GI symptoms were not worse when compared to those without any GI symptoms in our western study population. Importantly, we found a significantly high risk of developing ARDS requiring mechanical ventilation and CRS in COVID-19 patients with transaminitis at presentation. Notably, patients with an elevated AST on 
admission had a higher risk of mortality. Elevated total bilirubin anytime during hospitalization not only had a significantly higher likelihood of requiring mechanical ventilation but was also associated with higher mortality. Thus, frontline clinicians and first responders might consider evaluating for transaminitis in their preliminary laboratory assessments for making decisions regarding initial triaging and closer follow up. Prospective studies with a larger number of patients are required to further validate our observations.

\section{Additional Information \\ Disclosures}

Human subjects: Consent was obtained by all participants in this study. Animal subjects: All authors have confirmed that this study did not involve animal subjects or tissue. Conflicts of interest: In compliance with the ICMJE uniform disclosure form, all authors declare the following: Payment/services info: All authors have declared that no financial support was received from any organization for the submitted work. Financial relationships: All authors have declared that they have no financial relationships at present or within the previous three years with any organizations that might have an interest in the submitted work. Other relationships: All authors have declared that there are no other relationships or activities that could appear to have influenced the submitted work.

\section{References}

1. Verdecchia P, Cavallini C, Spanevello A, Angeli F: The pivotal link between ACE2 deficiency and SARS-CoV2 infection. Eur J Intern Med. 2020, 76:14-20. 10.1016/j.ejim.2020.04.037

2. Zhang H, Li H-B, Lyu J-R, et al.: Specific ACE2 expression in small intestinal enterocytes may cause gastrointestinal symptoms and injury after 2019-nCoV infection. Int J Infect Dis. 2020, 96:19-24. 10.1016/j.ijid.2020.04.027

3. Leung WK, To K, Chan PKS, et al.: Enteric involvement of severe acute respiratory syndrome-associated coronavirus infection. Gastroenterology. 2003, 125:1011-1017.

4. A case series of children with 2019 novel coronavirus infection: clinical and epidemiological features | Clinical Infectious Diseases | Oxford Academic. (2020). Accessed: May 12, 2020: https://academic.oup.com/cid/advance-article/doi/10.1093/cid/ciaa198/5766430.

5. Jin X, Lian JS, Hu JH, et al.: Epidemiological, clinical and virological characteristics of 74 cases of coronavirus-infected disease 2019 (COVID-19) with gastrointestinal symptoms. Gut. 2020, 69:1002-1009. 10.1136/gutjnl-2020-320926

6. Zhang C, Shi L, Wang FS: Liver injury in COVID- 19: management and challenges . Lancet Gastroenterol Hepatol. 2020, 5:428-430. 10.1016/S2468-1253(20)30057-1

7. Hajifathalian K, Krisko T, Mehta A, et al.: Gastrointestinal and hepatic manifestations of 2019 novel coronavirus disease in a large cohort of infected patients from New York: clinical implications. Gastroenterology. 2020, 159:1137.e2-1140.e2. 10.1053/i.gastro.2020.05.010

8. Mao R, Oiu Y, He J-S, et al.: Manifestations and prognosis of gastrointestinal and liver involvement in patients with COVID- 19: a systematic review and meta-analysis. Lancet Gastroenterol Hepatol. 2020, 5:667-678. 10.1016/S2468-1253(20)30126-6

9. Pan L, Mu M, Yang P, et al.: Clinical characteristics of COVID-19 patients with digestive symptoms in Hubei, China: a descriptive, cross-sectional, multicenter study. Am J Gastroenterol. 2020, 115:766-773. 10.14309/ajg.0000000000000620

10. Lin L, Jiang X, Zhang Z, et al.: Gastrointestinal symptoms of 95 cases with SARS-CoV-2 infection . Gut. 2020, 10.1136/gutjnl-2020-321013

11. Chen N, Zhou M, Dong X, et al.: Epidemiological and clinical characteristics of 99 cases of 2019 novel coronavirus pneumonia in Wuhan, China: a descriptive study. Lancet. 2020, 395:P507-P513.

12. Fan Z, Chen L, Jun L, et al.: Clinical features of COVID-19-related liver functional abnormality . Clin Gastroenterol Hepatol. 2020, 18:1561-1566. 10.1016/j.cgh.2020.04.002

13. Cai Q, Huang D, Ou P, et al.: COVID-19 in a designated infectious diseases hospital outside Hubei Province, China. Allergy Eur J Allergy Clin Immunol. 2020, 75:1742-1752. 10.1111/all.14309

14. Guan W-J, Ni Z-Y, Hu Y, et al.: Clinical characteristics of 2019 novel coronavirus infection in China . medRxiv. 2020, 10.1101/2020.02.06.20020974

15. Zhang B, Zhou X, Qiu Y, et al.: Clinical characteristics of 82 death cases with COVID-19 . medRxiv. 2020, 10.1101/2020.02.26.20028191

16. Wang D, Hu B, Hu C, et al.: Clinical characteristics of 138 hospitalized patients with 2019 novel coronavirus-infected pneumonia in Wuhan, China. J Am Med Assoc. 2020, 323:1061-1069. 10.1001/jama.2020.1585

17. Huang C, Wang Y, Li X, et al.: Clinical features of patients infected with 2019 novel coronavirus in Wuhan, China. Lancet. 2020, 395:497-506. 10.1016/S0140-6736(20)30183-5

18. Li J, Fan J-G: Characteristics and mechanism of liver injury in 2019 coronavirus disease . J Clin Transl Hepatol. 2020, 8:1-5. 10.14218/jcth.2020.00019

19. Li L, Li S, Xu M, et al.: The level of plasma C-reactive protein is closely related to the liver injury in patients with COVID-19. medRxiv. 2020,

20. Hoffmann M, Kleine-Weber H, Krueger N, Mueller MA, Drosten C, Poehlmann S: The novel coronavirus 2019 (2019-nCoV) uses the SARS-coronavirus receptor ACE2 and the cellular protease TMPRSS2 for entry into target cells. bioRxiv. 2020, 10.1101/2020.01.31.929042

21. Jiang F, Deng L, Zhang L, Cai Y, Cheung CW, Xia Z: Review of the clinical characteristics of coronavirus disease 2019 (COVID-19). J Gen Intern Med. 2020, 35:1545-1549. 10.1007/s11606-020-05762-w

22. Zaim S, Chong JH, Sankaranarayanan V, Harky A: COVID-19 and multiorgan response. Curr Probl Cardiol. 2020, 45:100618. 10.1016/j.cpcardiol.2020.100618 


\section{Cureus}

23. Xu Z, Shi L, Wang Y, et al.: Pathological findings of COVID-19 associated with acute respiratory distress syndrome. Lancet Respir Med. 2020, 8:420-422. 10.1016/S2213-2600(20)30076-X

24. Zhang C, Wu Z, Li JW, Zhao H, Wang GQ: The cytokine release syndrome (CRS) of severe COVID-19 and interleukin-6 receptor (IL-6R) antagonist Tocilizumab may be the key to reduce the mortality. Int J Antimicrob Agents. 2020, 55:105954. 10.1016/j.ijantimicag.2020.105954

25. Genovese MC, Kremer JM, van Vollenhoven RF, et al.: Transaminase levels and hepatic events during tocilizumab treatment: pooled analysis of long-term clinical trial safety data in rheumatoid arthritis. Arthritis Rheumatol. 2017, 69:1751-1761. 10.1002/art.40176 